\title{
Enhanced plasma miR-142-5p promotes the progression of intrahepatic cholangiocarcinoma via targeting PTEN
}

\author{
GUIFEN WEI, YITING YUAN, XINZHONG HE, LIMING JIN and DI JIN \\ Department of Hepatobiliary and Pancreatic Surgery, The First People's Hospital of Tongxiang, \\ Tongxiang, Zhejiang 314500, P.R. China
}

Received March 30, 2018; Accepted August 10, 2018

DOI: $10.3892 /$ etm.2019.7438

\begin{abstract}
The aim of the present study was to evaluate the expression and specific role of microRNA (miR)-142-5p in the progression of intrahepatic cholangiocarcinoma (ICC). Reverse transcription-quantitative polymerase chain reaction was performed to evaluate miR-142-5p expression in patients with ICC and healthy control subjects. The results revealed that plasma miR-142-5p was significantly increased in patients with ICC compared with the control group. Furthermore, miR-142-5p was also increased in ICC tissues compared with adjacent non-neoplastic tissues. Compared with patients with Ta-T1 stage ICC, miR-142-5p was significantly elevated in patients with ICC $\geq \mathrm{T} 2$ stage. Patients with ICC at G3 stage had much higher plasma miR-142-5p levels compared with those at G1/2 stage. Receiver operating characteristic analysis indicated that miR-142-5p could be used as a biomarker to differentiate patients with ICC from healthy controls. Kaplan-Meier analysis demonstrated that plasma miR-142-5p was negatively correlated with survival in patients with ICC. A dual luciferase reporter assay indicated that miR-142-5p significantly suppressed the relative luciferase activity of pmirGLO-PTEN-3' untranslated region compared with the control group. In summary, the results of the present study provide novel data indicating that plasma miR-142-5p is significantly upregulated in patients with ICC. miR-142-5p may therefore have potential as a biomarker for screening patients with ICC from healthy controls.
\end{abstract}

\section{Introduction}

Intrahepatic cholangiocarcinoma (ICC) is one of the most common hepatic malignancies worldwide (1), the incidence

Correspondence to: Dr Di Jin, Department of Hepatobiliary and Pancreatic Surgery, The First People's Hospital of Tongxiang, 1918 Wutong Street, Jiaochang East Road, Wutong, Tongxiang, Zhejiang 314500, P.R. China

E-mail: kaihui2018@126.com

Key words: microRNA-142-5p, intrahepatic cholangiocarcinoma, phosphate and tensin homolog, biomarker and mortality of which has increased in recent years (2). Previous studies have suggested that multiple signaling pathways are involved in the progression of $\operatorname{ICC}(3,4)$; however, the specific mechanisms underlying ICC etiology remain unknown. As cystoscopy is invasive and expensive, there is a need to identify potential diagnostic biomarkers for ICC in order to improve the early detection of $\operatorname{ICC}(5,6)$.

MicroRNAs (miRs) are small non-coding RNAs $\sim 22$ nucleotides in length that are associated with multiple biologic processes including cell proliferation, differentiation and apoptosis (7). Abnormal expression of miRNAs has been widely identified in different diseases $(8,9)$. For instance, miR-590-3p inhibits epithelial-mesenchymal transition in ICC via suppressing Smad interacting protein 1 expression (9). Additionally, several differentially expressed miRNAs have been reported as potential diagnostic biomarkers for patients with ICC; for instance, high miR-146a expression in the plasma and tumor tissues is reportedly associated with prolonged overall survival in surgical patients with ICC (7).

Abnormal miR-142-5p expression has been widely reported in different tumors (10-12). miR-142-5p acts as a tumor suppressor via targeting phosphatidylinositol-4,5-bisphosphate 3-kinase catalytic subunit $\alpha$ in non-small cell lung cancer (10). High miR-142-5p expression is also associated with the biological aggressiveness of colorectal cancer (11) and has been reported as a potential predictive biomarker for recurrence risk in patients with gastric cancer (12). The focus of the present study was miR-142-5p and its specific role in the progression of ICC. The aim was to evaluate the expression of miR-142-5p in ICC tissues and elucidate the potential underlying mechanism.

\section{Materials and methods}

Cell culture. Two hundred and ninety-three cells were obtained from the American Type Culture Collection (Manassas, VA, USA) and cultured in DMEM (Invitrogen; Thermo Fisher Scientific, Inc., Waltham, MA, USA) supplemented with $10 \%$ heat-inactivated fetal calf serum (Thermo Fisher Scientific, Inc.), $100 \mathrm{U} / \mathrm{ml}$ penicillin and streptomycin in $25-\mathrm{cm}^{2}$ culture flasks at $37^{\circ} \mathrm{C}$ in a humidified atmosphere containing $5 \% \mathrm{CO}_{2}$.

Patients and specimens. Human clinical samples were obtained from 100 patients with ICC between December 2016 
and November 2017 at The First People's Hospital of Tongxiang (Tongxiang, China). Corresponding adjacent, non-neoplastic tissues from the macroscopic tumor margin were isolated and used as controls. ICC diagnosis was based on criteria outlined by the World Health Organization (13) and tumor differentiation was based on the classification proposed by Edmondson and Steiner (13). The clinical classification of tumors was performed according to the 7 th edition of the tumor-node-metastasis classification system of the International Union Against Cancer (14). Patient characteristics are presented in Table I.

Patients were excluded from the current study if they exhibited: Failure of important organs, including the heart, lungs, kidneys and brain, intolerance to surgery, distant organ metastasis, lymph node involvement beyond the hepatoduodenal ligament, hilar or caval lymph nodes, preoperative chemotherapy or radiotherapy and preoperative liver treatment (arterial chemoembolization, radiofrequency ablation or percutaneous ethanol injection). All samples were immediately snap-frozen in liquid nitrogen and stored at $-80^{\circ} \mathrm{C}$. Whole blood samples were prospectively collected from patients with ICC and healthy controls without urologic malignancies. Whole blood $(5-8 \mathrm{ml})$ was collected in EDTA tubes and samples were centrifuged twice at $3,000 \mathrm{x} \mathrm{g}$ at $4^{\circ} \mathrm{C}$ for $15 \mathrm{~min}$. The plasma was then stored at $-80^{\circ} \mathrm{C}$. All research protocols were approved by The First People's Hospital of Tongxiang and written informed consent was obtained from all participants.

Plasma RNA isolation. Total RNA was isolated from whole blood samples using RNAVzol LS or tissue samples using or RNAVzol (Vigorous Biotechnology Beijing Co., Ltd., Beijing, China) according to the manufacturer's protocol. The quality, quantity and integrity of RNA were monitored using a NanoDrop spectrophotometer (ND-1000; Nanodrop; Thermo Fisher Scientific, Inc., Pittsburgh, PA, USA).

Reverse transcription-quantitative polymerase chain reaction $(R T-q P C R)$. A total of $1 \mu \mathrm{g}$ RNA was reverse transcribed using Moloney Murine Leukemia Virus reverse transcription enzyme (Applied Biosystems; Thermo Fisher Scientific, Inc.) with specific primers. The temperature protocol used for RT was as follows: $72^{\circ} \mathrm{C}$ for $10 \mathrm{~min} ; 42^{\circ} \mathrm{C}$ for $60 \mathrm{~min}$, $72^{\circ} \mathrm{C}$ for $5 \mathrm{~min}$ and $95^{\circ} \mathrm{C}$ for $2 \mathrm{~min}$. qPCR was performed using SYBR Green Supermix (Bio-Rad Laboratories, Inc., Hercules, CA, USA) in an iCycler iQ real-time PCR detection system. The PCR amplifications were performed in a $10 \mu \mathrm{l}$ reaction system containing $5 \mu \mathrm{l}$ SYBR Green Supermix, $0.4 \mu \mathrm{l}$ forward primer, $0.4 \mu \mathrm{l}$ reverse primer, $2.2 \mu \mathrm{l}$ double distilled $\mathrm{H}_{2} \mathrm{O}$ and $2 \mu \mathrm{l}$ template cDNA. Thermocycling conditions were as follows: $95^{\circ} \mathrm{C}$ for $10 \mathrm{~min}$ followed by 50 cycles of $95^{\circ} \mathrm{C}$ for $10 \mathrm{sec}, 55^{\circ} \mathrm{C}$ for $10 \mathrm{sec}, 72^{\circ} \mathrm{C}$ for $5 \mathrm{sec}$, $99^{\circ} \mathrm{C}$ for $1 \mathrm{sec}, 59^{\circ} \mathrm{C}$ for $15 \mathrm{sec}$ and $95^{\circ} \mathrm{C}$ for $1 \mathrm{sec}$, followed by cooling to $40^{\circ} \mathrm{C}$. Relative mRNA expression was normalized to U6 using the $2^{-\Delta \Delta \mathrm{Cq}}$ method (15). Primer sequences were as follows: miR-142-5p-RT, 5'-GTCGTATCCAGT GCAGGGTCCGAGGTATTCGCACTGGATACGACAGTA G-3'; U6-RT, 5'-GTCGTATCCAGTGCAGGGTCCGAG GTATTCGCACTGGATACGACAAAATG-3'; miR-142-5p, forward 5'-CATAAAGTAGAAAGCACTACT-3'; U6, forward 5'-GCGCGTCGTGAAGCGTTC-3'; and universal
Table I. Clinicopathological features of patients with ICC and healthy controls.

\begin{tabular}{lcc}
\hline Variables & Patients with ICC & Healthy controls \\
\hline Sex ratio (male/female) & $75 / 25$ & $39 / 11$ \\
Age & & \\
$\geq 60$ years & 64 & 35 \\
$<60$ years & 36 & 15 \\
Stage & & \\
Ta & 33 & - \\
T1 & 27 & - \\
T2 & 17 & - \\
T3 & 13 & - \\
T4 & 10 & - \\
Grade & & - \\
1 & 32 & - \\
2 & 38 & \\
3 & 30 & \\
\hline
\end{tabular}

ICC, intrahepatic cholangiocarcinoma.

reverse primer, 5'-GTGCAGGGTCCGAGGT-3'. The criteria for dividing low and high expression of miR-142-5p were determined as follows: Relative mRNA expression was normalized to U6 using the $2^{-\Delta \Delta \mathrm{Cq}}$ method (15). Patients with below average $2^{-\Delta \Delta C q}$ were classed as low miR-142-5p expression, while those with above average $2^{-\Delta \Delta \mathrm{Cq}}$ were classed as high miR-142-5p expression.

Transient transfection. A total of $6 \times 10^{5} 293$ cells were seeded in 6-well plates with $2 \mathrm{ml}$ DMEM supplemented with serum and antibiotics as above. miR-142-5p mimics, inhibitors, or miR negative controls (miR-NC; Shanghai GenePharma Co., Ltd., Shanghai, China) were mixed with HiperFect transfection reagent (Qiagen $\mathrm{GmbH}$, Hilden, Germany) and incubated at room temperature for $10 \mathrm{~min}$. This mixture was then added to cultured 293 cells for $48 \mathrm{~h}$. The interval between transfection and subsequent experimentation was $48 \mathrm{~h}$.

MiRNA target prediction and dual-luciferase reporter assay. TargetScan (https://www.targetscan.org) was used to predict potential target genes of miR-142-5p. The 3'-untranslated region (3'UTR) of phosphate and tensin homolog (PTEN) was cloned into the pmirGLO plasmid. After 293 cells were cultured for $24 \mathrm{~h}$ at $37^{\circ} \mathrm{C}$ in DMEM medium, miR-142-5p or scramble were cotransfected with blank pmirGLO or pmirGLO-PTEN-3'UTR using Vigofect (Vigorous Biotechnology Beijing Co., Ltd.) according to the manufacturer's protocol. Luciferase activity was analyzed using a Dual-Luciferase Reporter Assay System (E1910; Promega Corp., Madison, WI, USA).

Statistical analysis. Data are presented as the mean \pm standard error of the mean. Two-tailed unpaired Student's t-tests were used to compare two groups. Multiple group comparisons were made using one-way analysis of variance followed by 
A

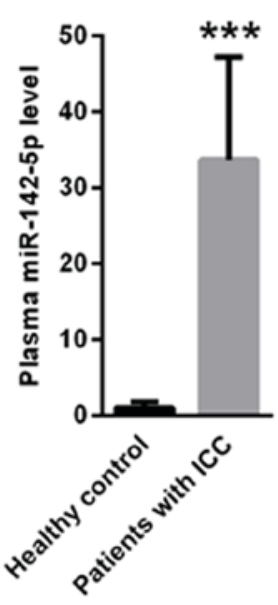

B

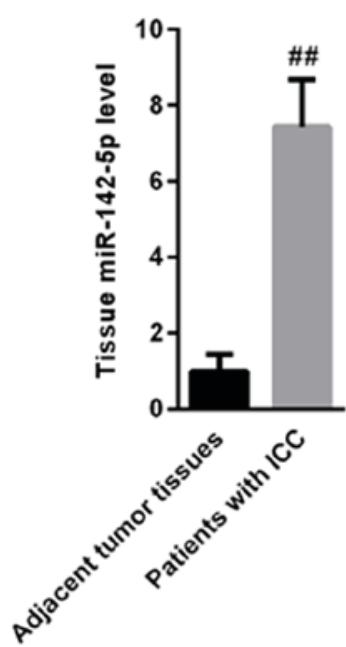

C

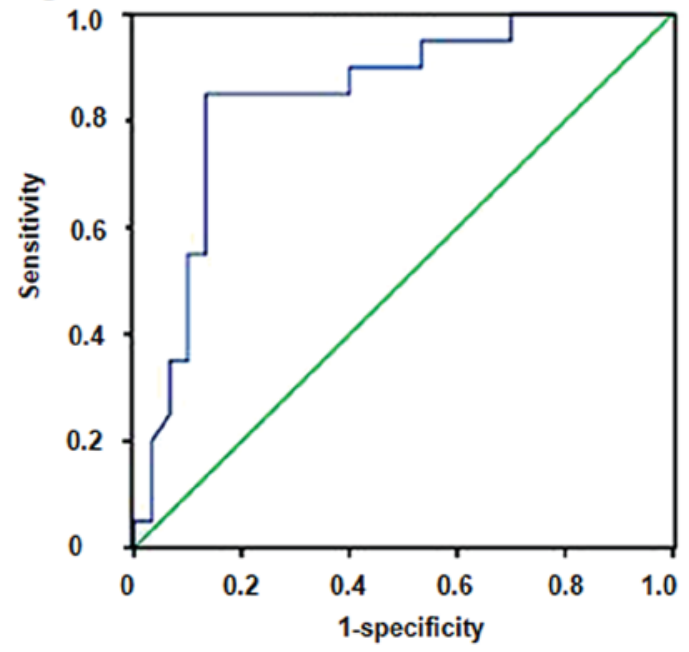

Figure 1. miR-142-5p expression in plasma and tissue samples. (A) Plasma miR-142-5p in healthy controls and patients with ICC. (B) miR-142-5p expression in tumor and adjacent non-neoplastic tissues. (C) Receiver operating characteristic analysis indicated that plasma miR-142-5p could screen patients with ICC from healthy controls. ${ }^{* * *} \mathrm{P}<0.001$ vs. control and ${ }^{\# \#} \mathrm{P}<0.01$ vs. adjacent non-neoplastic tissues. miR, microRNA; ICC, intrahepatic cholangiocarcinoma.

Table II. Multivariate Cox regression analysis for miR-142-5p in patients with intrahepatic cholangiocarcinoma.

\begin{tabular}{lccr}
\hline Parameter & Hazard ratio & 95\% confidence interval & P-value \\
\hline miR-142-5p & 3.508 & $1.783-6.968$ & $<0.001$ \\
Sex & 0.843 & $0.532-1.467$ & 0.486 \\
Age & 1.513 & $0.879-1.987$ & 0.114 \\
Tumor size & 1.021 & $0.621-1.831$ & 0.876 \\
Lymph node metastasis & 1.032 & $0.653-1.902$ & 0.821 \\
Clinical stage & 0.926 & $0.602-1.623$ & 0.821 \\
Histological type & 1.024 & $0.597-1.821$ & 0.921 \\
Differentiation & 1.067 & $0.672-1.906$ & 0.798 \\
\hline
\end{tabular}

miR, microRNA.

Tukey's multiple comparison test. Receiver operating characteristic curve (ROC) analysis was used to assess the efficacy of miR-142-5p as a biomarker. Kaplan-Meier survival analysis was also performed and survival differences were assessed using a log-rank test. Cox regression assay was performed to evaluate the prognostic value of miR-142-5p in patients with ICC. SPSS (version 20.0, SPSS, Inc., Chicago, IL, USA) was used for all statistical analysis. $\mathrm{P}<0.05$ was considered to indicate a statistically significant difference.

\section{Results}

Plasma and tissue miR-142-5p levels are upregulated in patients with ICC. Compared with healthy controls $(1 \pm 0.87)$, plasma miR-142-5p was significantly upregulated in patients with ICC (33.7 \pm 13.5 ; Fig. 1A). Furthermore, miR-142-5p expression was significantly higher in tumor tissues $(7.45 \pm 1.23)$ compared with adjacent non-neoplastic tissues $(1 \pm 0.45$; Fig. 1B). ROC analysis indicated that plasma miR-142-5p could be used to screen patients with ICC from healthy controls, with an area under the curve of 0.844 , (95\% confidence interval: 0.730-0.959; Fig. 1C). Furthermore, the prognostic value of miR-142-5p was assessed using Cox analysis. The results revealed that miR-142-5p overexpression was an independent prognostic factor for patients with ICC (hazard ratio 3.508, 95\% confidence interval 1.783-6.968; Table II).

miR-142-5p expression is positively correlated with ICC metastasis and invasion. Compared with patients with Ta-T1 stage ICC, $(1 \pm 0.68)$, plasma miR-142-5p was significantly elevated in patients with ICC with $\geq \mathrm{T} 2$ staging $(21.5 \pm 5.93$; Fig. 2A). Furthermore, patients with ICC at G3 stage had significantly higher plasma miR-142-5p levels (17.8 44.56$)$

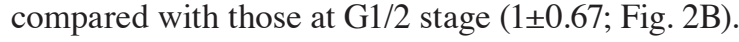

Plasma miR-142-5p is negatively correlated with survival in ICC cancer patients. The results of Kaplan-Meier analysis revealed that patients with high plasma miR-142-5p had a 
Table III. Clinicopathological features of patients with intrahepatic cholangiocarcinoma and healthy control subjects.

\begin{tabular}{|c|c|c|c|c|c|c|c|}
\hline \multirow[b]{2}{*}{ Variables } & \multirow[b]{2}{*}{$\mathrm{N}$} & \multicolumn{3}{|c|}{ miR-142-5p } & \multicolumn{3}{|c|}{ Overall survival, months } \\
\hline & & Low & High & P-value & Mean & $95 \% \mathrm{CI}$ & P-value \\
\hline \multicolumn{8}{|l|}{ Sex } \\
\hline Male & 75 & 37 & 38 & 0.985 & 44.67 & $39.05-51.94$ & 0.435 \\
\hline Female & 25 & 13 & 12 & & 38.25 & $32.56-43.15$ & \\
\hline \multicolumn{8}{|l|}{ Age } \\
\hline$\geq 60$ & 64 & 34 & 30 & 0.936 & 40.87 & $36.87-47.35$ & 0.098 \\
\hline$<60$ & 36 & 18 & 18 & & 42.86 & $31.45-50.87$ & \\
\hline \multicolumn{8}{|l|}{ Stage } \\
\hline $\mathrm{Ta}$ & 33 & 15 & 18 & 0.016 & 52.56 & $46.87-57.67$ & 0.012 \\
\hline $\mathrm{T} 1$ & 27 & 13 & 14 & & 46.53 & $39.75-53.12$ & \\
\hline $\mathrm{T} 2$ & 17 & 9 & 8 & & 36.54 & $30.57-43.22$ & \\
\hline $\mathrm{T} 3$ & 13 & 6 & 7 & & 29.35 & $27.01-34.52$ & \\
\hline $\mathrm{T} 4$ & 10 & 4 & 6 & & 20.91 & $5.78-32.87$ & \\
\hline \multicolumn{8}{|l|}{ Grade } \\
\hline 1 & 32 & 17 & 15 & 0.032 & 46.08 & $40.35-50.76$ & 0.009 \\
\hline 2 & 38 & 19 & 19 & & 36.45 & $30.23-43.12$ & \\
\hline 3 & 30 & 16 & 14 & & 21.34 & $6.56-35.96$ & \\
\hline
\end{tabular}

CI, confidence interval.
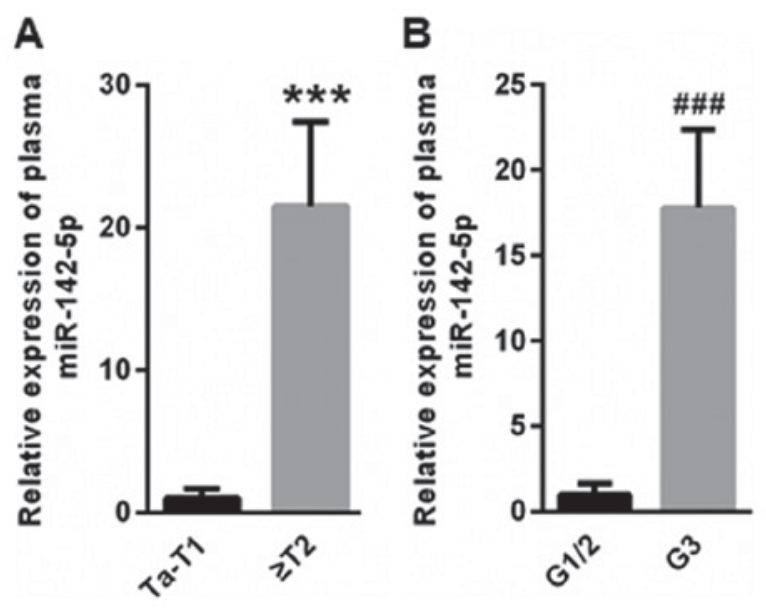

Figure 2. miR-142-5p is positively correlated with ICC metastasis and invasion. (A) Plasma miR-142-5p expression in patients with Ta-T1 and $\geq \mathrm{T} 2$ stage ICC. (B) Plasma miR-142-5p expression in patients with TG1/2 and G3 stage ICC. ${ }^{* * *} \mathrm{P}<0.001$ vs. Ta-T1 and ${ }^{\# \# \#} \mathrm{P}<0.001$ vs. G1/2. miR, microRNA; ICC, intrahepatic cholangiocarcinoma.

poorer survival rate compared with those with low plasma miR-142-5p, with 5-year overall survival rates of 27.86 and $51.46 \%$, respectively (Fig. 3). In addition, to analyze whether clinical factors, including sex, age, tumor diameter and tumor differentiation affect ICC prognosis, Kaplan-Meier survival curves were plotted and compared using a log-rank test (Table III). Log-rank analysis demonstrated that increased miR-142-5p was significantly correlated with tumor differentiation and malignancy (Table III).

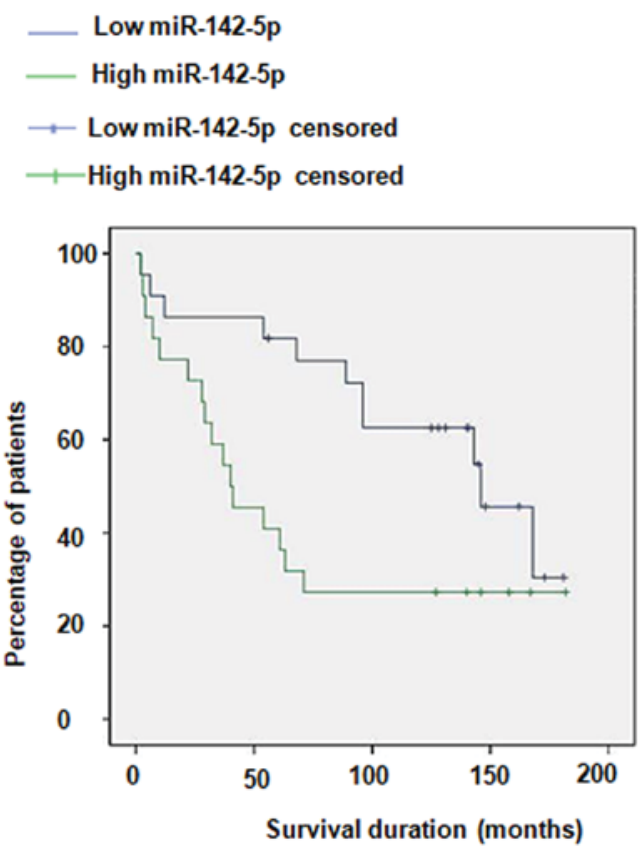

Figure 3. Kaplan-Meier analysis for plasma miR-142-5p expression and the survival of patients with intrahepatic cholangiocarcinoma. miR, microRNA.

PTEN is a target gene of miR-142-5p. Based on the results of TargetScan analysis, a conserved binding site of miR-142-5p in the 3'UTR of PTEN was identified (Fig. 4A). Dual luciferase reporter assay results indicated that miR-142-5p significantly suppressed the relative luciferase activity of pmirGLO-PTEN-3'UTR compared with the control (Fig. 4B). 
A

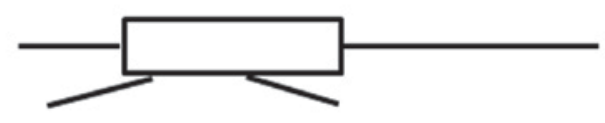

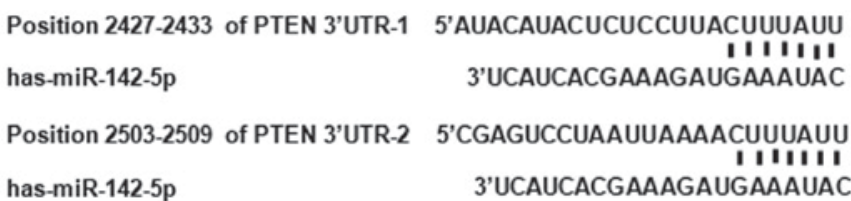
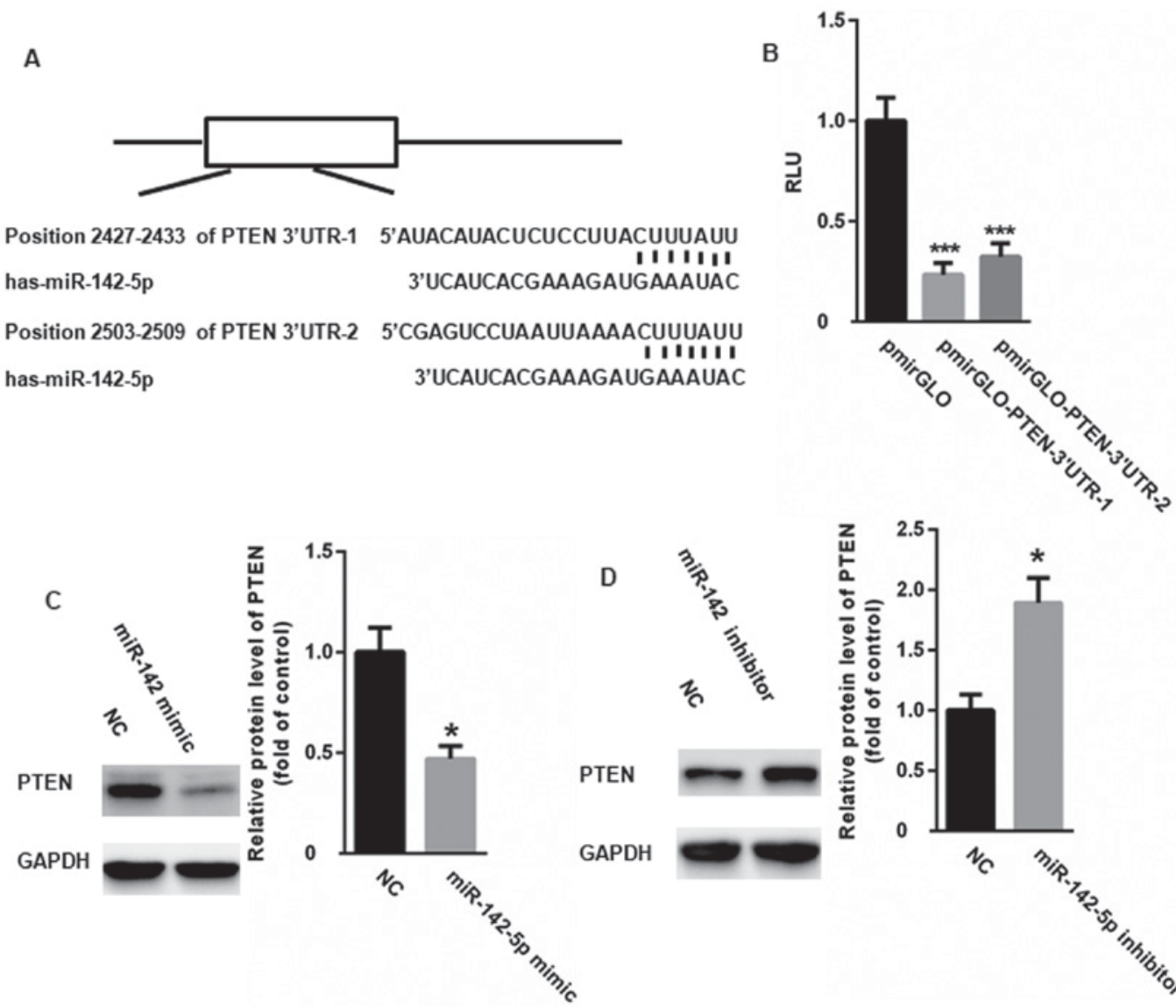

D

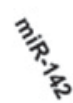

PTEN

GAPDH

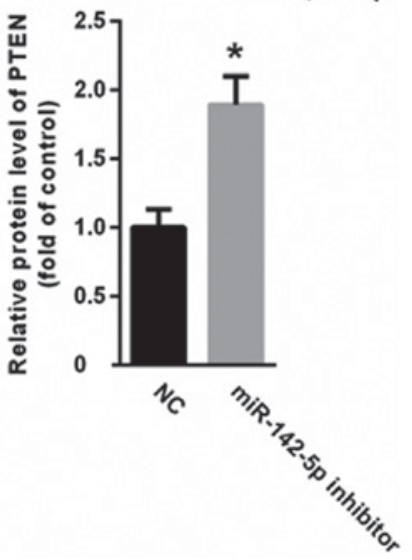

Figure 4. PTEN is a target gene of miR-142-5p. (A) A conserved binding site of miR-142-5p in the 3'UTR of PTEN was identified using TargetScan. (B) A dual luciferase reporter assay was performed to confirm the TargetScan prediction. Western blotting was performed to measure PTEN expression in cells transfected with (C) miR-142-5p mimics and (D) inhibitors. ${ }^{*} \mathrm{P}<0.05$ vs. miR-NC. ${ }^{* * *} \mathrm{P}<0.001$ vs. pmirGLO. RLU, relative luciferase unit; PTEN, phosphate and tensin homolog; miR, microRNA; 3'UTR, 3'-untranslated region.

Western blotting also revealed that miR-142-5p overexpression downregulated PTEN (Fig. 4C), while miR-142-5p knockdown resulted in PTEN upregulation (Fig. 4D). These results confirm PTEN as a target gene of miR-142-5p.

\section{Discussion}

ICC is the second most common intrahepatic primary tumor after hepatocellular carcinoma and ICC is highly invasive by nature and frequently metastasizes (16). It has been reported that chromosomal anomalies, genetic polymorphisms and genetic or epigenetic alterations may contribute to the tumorigenesis and progression of ICC $(17,18)$. It is important to detect ICC early in order to improve treatment outcomes. Increasing evidence has suggested that miRNAs may be used as potential diagnostic biomarkers for ICC and may also serve as therapeutic targets $(19,20)$.

miRNAs are able to stably exist in bodily fluids, including serum, plasma, saliva, urine and tears $(20,21)$. Furthermore, miRNAs can be easily detected in small amounts and are resistant to degradation $(20,22)$. These characteristics make miRNAs attractive as potential biomarkers (20). In the present study it was determined that data showed that plasma miR-142-5p was significantly increased in patients with ICC compared with healthy controls. Furthermore, miR-142-5p levels were increased in ICC tumor tissues compared with adjacent non-neoplastic tissues. Further analysis revealed a positive correlation between miR-142-5p and clinical outcome. Compared with patients with ICC at Ta-T1 stage, miR-142-5p was significantly upregulated in patients with ICC at $\geq \mathrm{T} 2$ stage. Additionally, patients with ICC at G3 stage had higher plasma miR-142-5p levels compared with those at G1/2 stage. These data indicate that miR-142-5p expression is positively correlated with therapy and outcome. ROC analysis indicated that miR-142-5p could be used to differentiate patients with ICC from healthy controls. Additionally, Kaplan-Meier analysis revealed that plasma miR-142-5p is negatively correlated with survival in patients with ICC. These data indicate that plasma miR-142-5p may be useful for the early detection of cancer and individualized therapies. The main focus of the present study was PTEN, which is an important tumor suppressor in the development of ICC (23). Mutation and genomic loss of PTEN have been widely reported in a number of cancers $(24,25)$. It has been also demonstrated that liver-specific deletion of PTEN in a mouse model results in the development of ICC $(26,27)$. As an important tumor suppressor, PTEN mainly acts to dephosphorylate phosphatidylinositol-3,4,5-trisphosphate $[\operatorname{Ptd} \operatorname{Ins}(3,4,5)$ P3], which potently activates 3-phosphoinositide-dependent kinase (PDK) and protein kinase B (AKT) (28). However, PTEN loss leads to excessive recruitment of PtdIns(3,4,5)P3 at the plasma membrane, thereby activating a subset of proteins, including the AKT family and PDK1 (28). AKT signaling 
induces cell survival, cell proliferation, angiogenesis and cellular metabolism via phosphorylating downstream signaling proteins $(29,30)$. The results of the present study revealed that PTEN was a target gene of miR-142-5p. PTEN is widely acknowledged as a tumor suppressor that is mutated in multiple tumors (31-33). In the progression of ICC cancer, PTEN could negatively regulate the AKT/PKB signaling pathway, thereby increasing cancer cell growth and survival $(34,35)$. The results of the present study indicate that miR-142-5p may suppress PTEN expression, thereby resulting in the malignant proliferation and increased viability of cancer cells.

In summary, this study presents novel data indicating that plasma miR-142-5p is significantly upregulated in patients with ICC. Further analysis demonstrated that plasma miR-142-5p could be used to screen patients with ICC from healthy controls by targeting PTEN. However, only limited samples were included in the current study. Thus, in further study, it may be necessary to include more patients to validate the application of miR-142-5p as a potential biomarker.

\section{Acknowledgements}

Not applicable.

\section{Funding}

The present study was supported by the Guided Science And Technology Program Of Tongxiang City (grant nos. 81700765 and 81570789), the Zhejiang Provincial Natural Science Foundation of China (grant no. LY14H030006), the Foundation for Young Scientists of Zhejiang Province Traditional Chinese Medicine (grant no. 2011ZQ008) and the Health and Family Planning Committee of Zhejiang Province (grant no. 2012KYB143).

\section{Availability of data and materials}

The datasets used and/or analyzed in the present study are available from the corresponding author on reasonable request.

\section{Authors' contributions}

GW performed the experiments and analyzed the data. YY, XH and LJ performed RT-qPCR experiments. DJ designed all experiments, analyzed the data and gave final approval of the version of the manuscript to be published. All authors read and approved the final manuscript.

\section{Ethics approval and consent to participate}

The research protocols were approved by the First People's Hospital of Tongxiang (Tongxiang, China) and written informed consent was obtained from the participants.

\section{Patient consent for publication}

Not applicable.

\section{Competing interests}

The authors declare that they have no competing interests.

\section{References}

1. Hu J, Chen FY, Zhou KQ, Zhou C, Cao Y, Sun HC, Fan J, Zhou J and Wang Z: Intrahepatic cholangiocarcinoma patients without indications of lymph node metastasis not benefit from lymph node dissection. Oncotarget 8: 113817-113827, 2017.

2. Ahn DH and Bekaii-Saab T: Biliary cancer: Intrahepatic cholangiocarcinoma vs. extrahepatic cholangiocarcinoma vs. gallbladder cancers: Classification and therapeutic implications. J Gastrointest Oncol 8: 293-301, 2017.

3. Bagante F, Spolverato G, Weiss M, Alexandrescu S, Marques HP, Aldrighetti L, Maithel SK, Pulitano C, Bauer TW, Shen F, et al: Impact of morphological status on long-term outcome among patients undergoing liver surgery for intrahepatic cholangiocarcinoma. Ann Surg Oncol 24: 2491-2501, 2017.

4. Bhalla A, Mann SA, Chen S, Cummings OW and Lin J: Histopathological evidence of neoplastic progression of von meyenburg complex to intrahepatic cholangiocarcinoma. Hum Pathol 67: 217-224, 2017.

5. Chang ME, Lei HJ, Chen MH, Yeh YC, Li CP, Hung YP, Hsia CY, Liu CA, Chau GY and Chao Y: Evaluation of prognostic factors and implication of lymph node dissection in intrahepatic cholangiocarcinoma: 10-year experience at a tertiary referral center. J Chin Med Assoc 80: 140-146, 2017.

6. Chen Y, Liu D, Liu P, Chen Y, Yu H and Zhang Q: Identification of biomarkers of intrahepatic cholangiocarcinoma via integrated analysis of mRNA and miRNA microarray data. Mol Med Rep 15: 1051-1056, 2017.

7. Zhang RX, Zheng Z, Li K, Wu XH and Zhu L: Both plasma and tumor tissue miR-146a high expression correlates with prolonged overall survival of surgical patients with intrahepatic cholangiocarcinoma. Medicine (Baltimore) 96: e8267, 2017.

8. Zhang S, Xiao J, Chai Y, Du YY, Liu Z, Huang K, Zhou X and Zhou W: LncRNA-CCAT1 promotes migration, invasion, and EMT in intrahepatic cholangiocarcinoma through suppressing miR-152. Dig Dis Sci 62: 3050-3058, 2017.

9. Zu C, Liu S, Cao W, Liu Z, Qiang H, Li Y, Cheng C, Ji L, Li J and Li J: MiR-590-3p suppresses epithelial-mesenchymal transition in intrahepatic cholangiocarcinoma by inhibiting SIP1 expression. Oncotarget 8: 34698-34708, 2017.

10. Wang Z, Liu Z, Fang X and Yang H: MiR-142-5p suppresses tumorigenesis by targeting PIK $3 \mathrm{CA}$ in non-small cell lung cancer. Cell Physiol Biochem 43: 2505-2515, 2017.

11. Islam F, Gopalan V, Vider J, Lu CT and Lam AK: MiR-142-5p act as an oncogenic microRNA in colorectal cancer: Clinicopathological and functional insights. Exp Mol Pathol 104: 98-107, 2018

12. Zhang X, Yan Z, Zhang J, Gong L, Li W, Cui J, Liu Y, Gao Z, Li J, Shen L and Lu Y: Combination of hsa-miR-375 and hsa-miR-142-5p as a predictor for recurrence risk in gastric cancer patients following surgical resection. Ann Oncol 22: 2257-2266, 2011.

13. Wittekind C: Pitfalls in the classification of liver tumors. Pathologe 27: 289-293, 2006 (In German).

14. Edge SB and Compton CC: The american joint committee on cancer: The 7th edition of the AJCC cancer staging manual and the future of TNM. Ann Surg Oncol 17: 1471-1474, 2010.

15. Livak KJ and Schmittgen TD: Analysis of relative gene expression data using real-time quantitative PCR and the 2(-Delta Delta C(T)) method. Methods 25: 402-408, 2001.

16. Bartella I and Dufour JF: Clinical diagnosis and staging of intrahepatic cholangiocarcinoma. J Gastrointestin Liver Dis 24: 481-489, 2015.

17. Rahnemai-Azar AA, Weisbrod A, Dillhoff M, Schmidt C and Pawlik TM: Intrahepatic cholangiocarcinoma: Molecular markers for diagnosis and prognosis. Surg Oncol 26: 125-137, 2017.

18. Pawlik TM: Intrahepatic cholangiocarcinoma: From diagnosis to treatment. Hepatobiliary Surg Nutr 6: 1, 2017.

19. Wang S, Yin J, Li T, Yuan L, Wang D, He J, Du X and Lu J: Upregulated circulating miR-150 is associated with the risk of intrahepatic cholangiocarcinoma. Oncol Rep 33: 819-825, 2015.

20. Correa-Gallego C, Maddalo D, Doussot A, Kemeny N, Kingham TP, Allen PJ, D'Angelica MI, DeMatteo RP, Betel D, Klimstra D, et al: Circulating plasma levels of MicroRNA-21 and MicroRNA-221 are potential diagnostic markers for primary intrahepatic cholangiocarcinoma. PLoS One 11: e0163699, 2016.

21. Cheng Q, Feng F, Zhu L, Zheng Y, Luo X, Liu C, Yi B and Jiang X: Circulating miR-106a is a novel prognostic and lymph node metastasis indicator for cholangiocarcinoma. Sci Rep 5: 16103, 2015. 
22. Yuan R, Wang G, Xu Z, Zhao H, Chen H, Han Y, Wang B, Zhou J, $\mathrm{Hu} \mathrm{H}$, Guo Z, et al: Up-regulated circulating miR-106a by DNA methylation promised a potential diagnostic and prognostic marker for gastric cancer. Anticancer Agents Med Chem 16: 1093-1100, 2016.

23. Zhou GY, Pan CW, Jin LX, Zheng JJ and Yi YX: Neoalbaconol inhibits cell growth of human cholangiocarcinoma cells by up-regulating PTEN. Am J Transl Res 8: 496-505, 2016.

24. Horie Y, Suzuki A, Kataoka E, Sasaki T, Hamada K, Sasaki J, Mizuno K, Hasegawa G, Kishimoto H, Iizuka M, et al: Hepatocyte-specific pten deficiency results in steatohepatitis and hepatocellular carcinomas. J Clin Invest 113: 1774-1783, 2004.

25. Trimboli AJ, Cantemir-Stone CZ, Li F, Wallace JA, Merchant A, Creasap N, Thompson JC, Caserta E, Wang H, Chong JL, et al: Pten in stromal fibroblasts suppresses mammary epithelial tumours. Nature 461: 1084-1091, 2009.

26. Xu X, Kobayashi S, Qiao W, Li C, Xiao C, Radaeva S, Stiles B, Wang RH, Ohara N, Yoshino T, et al: Induction of intrahepatic cholangiocellular carcinoma by liver-specific disruption of Smad4 and pten in mice. J Clin Invest 116: 1843-1852, 2006.

27. Marsh V, Davies EJ, Williams GT and Clarke AR: PTEN loss and KRAS activation cooperate in murine biliary tract malignancies. J Pathol 230: 165-173, 2013.

28. Chung JY, Hong SM, Choi BY, Cho H, Yu E and Hewitt SM The expression of phospho-AKT, phospho-mTOR, and PTEN in extrahepatic cholangiocarcinoma. Clin Cancer Res 15: 660-667, 2009.

29. Lee YR, Chen M and Pandolfi PP: The functions and regulation of the PTEN tumour suppressor: New modes and prospects. Nat Rev Mol Cell Biol 19: 547-562, 2018.
30. Song MS, Salmena L and Pandolfi PP: The functions and regulation of the PTEN tumour suppressor. Nat Rev Mol Cell Biol 13 283-296, 2012

31. Rimawi MF, De Angelis C, Contreras A, Pareja F, Geyer FC, Burke KA, Herrera S, Wang T, Mayer IA, Forero A, et al: Low PTEN levels and PIK3CA mutations predict resistance to neoadjuvant lapatinib and trastuzumab without chemotherapy in patients with HER2 over-expressing breast cancer. Breast Cancer Res Treat 167: 731-740, 2018.

32. Malaney P, Palumbo E, Semidey-Hurtado J, Hardee J, Stanford K, Kathiriya JJ, Patel D, Tian Z, Allen-Gipson D and Davé V: PTEN Physically interacts with and regulates E2F1-mediated transcription in lung cancer. Cell Cycle 17: 947-962, 2018.

33. Bian X, Gao J, Luo F, Rui C, Zheng T, Wang D, Wang Y, Roberts TM, Liu P, Zhao JJ and Cheng H: PTEN deficiency sensitizes endometrioid endometrial cancer to compound PARP-PI3K inhibition but not PARP inhibition as monotherapy. Oncogene 37: 341-351, 2018.

34. Ikenoue T,Terakado Y,NakagawaH,Hikiba Y,FujiiT,MatsubaraD, Noguchi R, Zhu C, Yamamoto K, Kudo Y, et al: Corrigendum: A novel mouse model of intrahepatic cholangiocarcinoma induced by liver-specific Kras activation and Pten deletion. Sci Rep 7: 39567, 2017.

35. Ikenoue T, Terakado Y, Nakagawa H, Hikiba Y, Fujii T, Matsubara D, Noguchi R, Zhu C, Yamamoto K, Kudo Y, et al: A novel mouse model of intrahepatic cholangiocarcinoma induced by liver-specific Kras activation and Pten deletion. Sci Rep 6: 23899, 2016. 